\title{
La prevenzione della nefrolitiasi: evidenze attuali e possibili miglioramenti metodologici nei trials futuri
}

Commento all'articolo: "Medical management to prevent recurrent nephrolithiasis in adults: a systematic review for an American College of Physicians Clinical Guideline”

\section{Salvatore Campo}

\author{
Responsabile Nazionale Area Urologica della Società Italiana di Medicina Generale (SIMG)
}

La nefrolitiasi è una condizione molto frequente, interessa circa il 13\% degli uomini e il $7 \%$ delle donne. Ha inferenze con il benessere in merito agli episodi dolorosi da colica e al possibile danno biologico sull'apparato urinario. È causa di costi economici, sia sanitari sia indiretti (perdita di giornate lavorative, di produttività ecc.) e in Italia, assieme all'insufficienza renale cronica e all'ipertrofia prostatica benigna, è una delle tre più frequenti cause di ricovero ospedaliero per motivi nefrourologici (1). Chi è sintomatico per un calcolo di Oxca ha la probabilità di farne un altro entro i 5 anni in una percentuale dal $35 \%$ al $50 \%$, in assenza di uno specifico trattamento (2). Gli "stone formers", per tutta la vita, debbono attendersi coliche, procedure terapeutiche, procedure interventistiche e sequele, con la frequente frustrazione della recidiva in pazienti che spesso sono stati educati alla profilassi ed hanno tenuto un comportamento consequenziale.

Questi aspetti hanno spinto la ricerca verso il conseguimento di ottimali strategie di prevenzione per una condizione la cui evoluzione risente della multifattorialità che la può sottendere e delle molteplici patologie che possono influenzarla.

La review presentata da Fink et al ha incluso 28 RCTs che hanno valutato l'efficacia e i rischi delle terapie dietetiche (8 trials) e farmacologiche (20 trials) nella prevenzione della nefrolitiasi (3). Per quanto concerne i comportamenti dietetici, l'aumento dell'assunzione idrica in maniera tale da determinare una diuresi di 2-2.5 1/die, la riduzione dell'assunzione dei soft-drink, un alto consumo di fibre e un minor consumo di proteine animali possono diminuire il rischio di recidiva litiasica. Per il ruolo della terapia farmacologica, 6 lavori hanno dimostrato l'efficacia dei diuretici tiazidici, altri 6 testimoniano a favore del ruolo dei citrati nel prevenire la formazione di calcoli di ossalato di calcio; 4 lavori hanno valutato l'efficacia dell'allopurinolo con l'inserimento di alcune variabili (quantità d'acqua introdotta e uso di bicarbonato di sodio per alcalinizzare le urine) che ne limitano l'estensibilità delle indicazioni relativamente al singolo trattamento. Gli Autori manifestano il dubbio che nella loro review la selezione degli studi, mediata dai principali e dedicati motori di ricerca web, potesse essere affetta da publication-bias.

Complessivamente, emergono sulla prevenzione della nefrolitiasi evidenze scientifiche già note da tempo ma non possono essere sottaciuti alcuni vulnus di tipo metodologico:

a. Nessuno dei RCTs presi in considerazione ha studiato il ruolo della prevenzione in funzione della composizione dei calcoli o del profilo biochimico urinario. Generalmente, i trials arruolano pazienti adulti con nefrolitiasi, almeno inizialmente idiopatica, senza correlare le caratteristiche biologiche con il singolo intervento preventivo; ne deriva che le indicazioni non sono estensibili a tutte le condizioni biologiche; si considerino le influenze dell'età, del genere, nello stesso genere delle diverse fasi del percorso di vita, delle co-morbidità che possono influenzare la litogenicità e le complicanze, ecc.

b. Nessuno dei trials ha comparato l'efficacia dei tiazidici, dei citrati e dell'allopurinolo tra di loro e non vi sono forti evidenze che l'aggiunta di citrati o di allopurinolo ai tiazidici determini un vantaggio aggiuntivo.

c. Molti trials non riportano eventuali rischi.

d. Pochi trials hanno come obiettivo principale la recidiva di nefrolitiasi sintomatica piuttosto che quella radiologica o ecografica.

e. Non è chiaro il tempo da assegnare ai diversi trattamenti preventivi perché si rivelino efficaci.

In un solo studio l'arruolamento è avvenuto nel setting delle cure primarie.

È auspicabile, per conseguire una maggiore chiarezza sulla problematica della prevenzione della nefrolitiasi, che gli studi futuri:

1. si pongano come obiettivo primario la prevenzione della litiasi sintomatica;

2. selezionino i soggetti arruolati sulla base del genere, dell'età e del profilo biochimico delle urine e dei calcoli;

3. valutino l'efficacia dei trattamenti singoli o in associazione in funzione della tipologia dei calcoli e dei tempi di trattamento; 
4. tengano in considerazione le eventuali co-morbidità esistenti;

5. operino l'arruolamento in un setting territoriale piuttosto che ospedaliero o in Unità specialistiche di riferimento per evitare possibili bias di selezione che finirebbero per limitare l'estensibilità dei risultati.

La scelta delle strategie suddette potrebbe conseguire risultati scientificamente più validi, rendere più chiaro il profilo di ogni intervento preventivo, singolo o in associazione, e condizionare positivamente la trasferibilità dei dati dei trial nella pratica clinica.

Dichiarazione di conflitto di interesse: L'Autore dichiara di non avere conflitto di interessi.

Contributi economici agli Autori: L'Autore dichiara di non avere ricevuto sponsorizzazioni economiche per la preparazione dell'articolo.

Indirizzo dell'Autore:

Dr. Salvatore Campo

Responsabile Nazionale Area Urologica della Società Italiana di Medicina Generale (SIMG)

campo.salva@tin.it

\section{Bibliografia}

1. Ministero della Salute. http://www.ministerosalute. it/programmazione/sdo/ric informazioni/sceltadia.jsp)

2. Goldfarb DS. In the clinic. Nephrolithiasis. American College of Physicians. Ann Inter Med 2009; 151: ITC2-1. www.annals.org/search.dtl

3. Fink HA, Wilt TJ, Eidman KE, et al. Medical management to prevent recurrent nephrolithiasis in adults: a systematic review for an American College of Physicians Clinical Guideline. Ann Intern Med 2013; 158: 535-43. 